\title{
Como a Tecnologia Assistiva tem auxiliado o Processo de Ensino/Aprendizagem? Mapeamento Sistemático a partir dos Trabalhos Publicados no SBIE
}

\author{
Lilian O. Ramires, Ellen P.R. Souza, Jonathan N. de Souza, Marcelo R. Freire, \\ Marcus V.S. Janurio, Ricardo E.F. Silva, Rostan S.F. Gomes, Tiago A.B. de Lima \\ Universidade Federal Rural de Pernambuco (UFRPE) \\ Caixa Postal 063 - CEP: 56.900-000 - Serra Talhada - PE - Brazil \\ \{liliramires, jonathan_zl9\}@hotmail.br; ellen.polliana, \\ celinho.freire, viniciussouza17, ricardo.emanuel66, rostan.ferreira, \\ tiagocalumbi\}@gmail.com
}

\begin{abstract}
Background: Assistive Technology (AT) is the technology used to identify services and resources used to increase, maintain, or improve functional capabilities of individuals with disabilities or reduced mobility. Aim: this works proposes a mapping and publicizing of AT resources applied to the teaching/learning process. Method: it was performed a Systematic Literature Mapping from Brazilian Symposium on Informatics in Education collection. Results: from 1149 primary studies analyzed, 79 were included in this research, hearing deficiency has the highest number of AT feature while speech has the lowest.
\end{abstract}

Resumo. Introdução: Tecnologia Assistiva (TA) é o termo utilizado para identificar os recursos e serviços que contribuem para proporcionar ou ampliar habilidades funcionais da $\mathrm{PcD}$ ou com mobilidade reduzida. Objetivo: este trabalho propõe a catalogação e divulgação dos recursos de TA que auxiliam as $P c D$, incapacidades ou mobilidade reduzida no processo de ensino/aprendizagem. Método: foi realizado um Mapeamento Sistemático a partir das publicações do Simpósio Brasileiro de Informática na Educação (SBIE). Resultados: dos 1149 estudos primários analisados, 79 foram incluídos neste estudo, a deficiência de audição aparece com o maior número de recurso de TA enquanto a de fala possui o menor.

\section{Introdução}

De acordo com o último censo do $\mathrm{IBGE}^{1}$, o número de Pessoas com Deficiência (PcD) chega a 45,6 milhões, representado um percentual de 23,9\% da população brasileira. Deste total, 67,2\% são pessoas com mais de 65 anos de idade. Há vários tipos de deficiência ou limitação, em diferentes níveis de severidade e combinações e podem ser temporárias ou definitivas. A deficiência visual aparece com o maior número de pessoas (35.791.488), seguida pela deficiência motora (13.273.969), auditiva (9.722.163) e, por fim, as deficiências mental e intelectual (2.617.025).

A Tecnologia Assistiva (TA) é um termo relativamente novo, utilizado para identificar os recursos e serviços que contribuem para proporcionar ou ampliar habilidades funcionais da PcD ou com mobilidade reduzida, com o propósito de promover inclusão e independência destes.

\footnotetext{
${ }^{1}$ Censo demográfico de 2010. As pessoas incluídas em mais de um tipo de deficiência foram contadas apenas uma vez.
} 
Dentre os recursos de TA, estão aqueles que auxiliam no processo de ensinoaprendizagem, possibilitando, não somente a inclusão, mas um melhor aprendizado e interação com os demais indivíduos. Segundo Bersch (2008), a TA deve ser entendida como o "recurso do usuário" e não como "recurso do profissional”. Esta característica diferencia a TA de outras tecnologias como a tecnologia educacional.

Neste sentido, através da cartilha intitulada "O Acesso de Alunos com Deficiência às Escolas e Classes Comuns da Rede Regular", o Ministério Público (2004) caracteriza o atendimento educacional especializado como aquilo que é necessariamente diferente do ensino escolar para melhor atender às especificidades dos alunos com deficiência, incluindo, principalmente, os instrumentos necessários à eliminação de barreiras que as pessoas com deficiência têm para relacionarem-se com o ambiente externo, tais como: o ensino da língua brasileira de sinais (LIBRAS), do código braile, o uso de recursos de informática e outras ferramentas tecnológicas.

Percebe-se, então, que a TA transcende à atuação restrita da saúde e/ou reabilitação, inserindo-se também no campo da educação. Assim, visando conhecer, catalogar e divulgar os recursos de TA que auxiliam as PcD, incapacidades ou mobilidade reduzida, este trabalho propõe um Mapeamento Sistemático sobre o uso de TA no processo de ensino/aprendizagem, a partir das publicações do Simpósio Brasileiro de Informática na Educação (SBIE). Os recursos de TA analisados neste trabalho são aqueles baseados em hardware e/ou software.

O restante do artigo está organizado da seguinte forma: a Seção 2 apresenta o referencial teórico sobre a área de TA e estudos secundários. A Seção 3 contém a metodologia utilizada para a realização do mapeamento. Na Seção 4 são descritos os resultados obtidos. A Seção 5 apresenta a discussão dos resultados. Por fim, a Seção 6 contém as conclusões e trabalhos futuros.

\section{Referencial Teórico}

O embasamento teórico para este trabalho fundamentasse em técnicas de realização de estudos secundários, conceitos e características das tecnologias assistivas.

\subsection{Estudos Secundários}

Diversos estudos secundários têm sido desenvolvidos com o propósito de buscar evidências a respeito de vários campos de estudo. Uma pesquisa secundária envolve a análise, avaliação e interpretação de resultados de estudos primários relacionados a um tópico em específico [Kitchenham 2004].

Segundo Dyba et al (2005), a revisão sistemática é um método de pesquisa utilizado para identificar, avaliar e sintetizar todos os estudos significativos, realizados sobre um tema específico. Este método deve ser rigoroso o suficiente para que possa ser replicado posteriormente e tem como propósito encontrar evidências sobre as perguntas de pesquisa ou lacunas que ainda precisam ser pesquisadas. Segundo Cavalcanti e Silva (2011), a literatura sobre metodologia científica distingue dois tipos de revisões:

- As Revisões Sistemáticas (RS) de literatura, que agregam resultados sobre a efetividade de um estudo, respondendo as perguntas de pesquisa do tipo: a intervenção $\boldsymbol{I}$ na população $\boldsymbol{P}$ é mais efetiva para obter o resultado $\boldsymbol{O}$ em um contexto $\boldsymbol{C}$ do que uma intervenção comparável $\boldsymbol{C}$ ? Resultando na estrutura 


\section{PICOC (Population, Intervention, Comparison, Outcome, Context)}

- Mapeamentos Sistemáticos (MS) ou estudos de escopo, cujo objetivo é identificar e analisar de forma geral toda a pesquisa em um tópico ou disciplina, respondendo a questões mais amplas relacionadas a tendências, como por exemplo: $\mathrm{O}$ que sabemos sobre um tópico $\boldsymbol{T}$ ?

Neste artigo foi utilizada a metodologia de MS com o propósito de catalogar e divulgar os recursos de TA que auxiliam as PcD, incapacidades ou mobilidade reduzida no processo de ensino/aprendizagem, a partir das publicações do SBIE.

\subsection{Tecnologia Assistiva}

Em 2006, a Secretaria Especial dos Direitos Humanos da Presidência da República (SEDH/PR) instituiu o Comitê de Ajudas Técnicas (CAT) que, dentre seus objetivos, tem a missão de apresentar propostas de políticas governamentais e parcerias entre a sociedade civil e órgãos públicos referentes à área de TA. Em 2007, o CAT propôs uma definição brasileira e mais ampla para TA, apresentado-a como "área do conhecimento, de característica interdisciplinar, que engloba produtos, recursos, metodologias, estratégias, práticas e serviços que objetivam promover a funcionalidade, relacionada à atividade e participação, de pessoas com deficiência, incapacidades ou mobilidade reduzida, visando sua autonomia, independência, qualidade de vida e inclusão social".

Os recursos de TA são organizados ou classificados de acordo com objetivos funcionais a que se destinam [CAT 2009]. A classificação dos recursos de TA adotada neste trabalho foi proposta pela pesquisadora brasileira Rita Bersh (2008). Ela leva em consideração as classificações da ISO 9999 (2007), a Classificação Nacional de Tecnologia Assistiva (2000) do Departamento de Educação dos Estados Unidos, o modelo de classificação Horizontal European Activities in Rehabilitation Technology (HEART) (1998) e a formação obtida pela autora no Programa de Certificação em Aplicações da Tecnologia Assistiva - ATACP do College of Extended Learning and Center on Disabilities, da California State University de Northridge.

\section{Metodologia}

O protocolo de pesquisa foi formulado de acordo com Kitchenham (2004) e Biolchini et al (2005). O Quadro 1 ilustra as etapas para realização do mapeamento, iniciando pela construção do protocolo, a execução e a análise, com o fim de divulgar os resultados mapeados. O detalhamento de cada um destes passos e seus subpassos é apresentado nas subseções que se seguem.

\section{Quadro 1. Etapas do Mapeamento Sistemático}

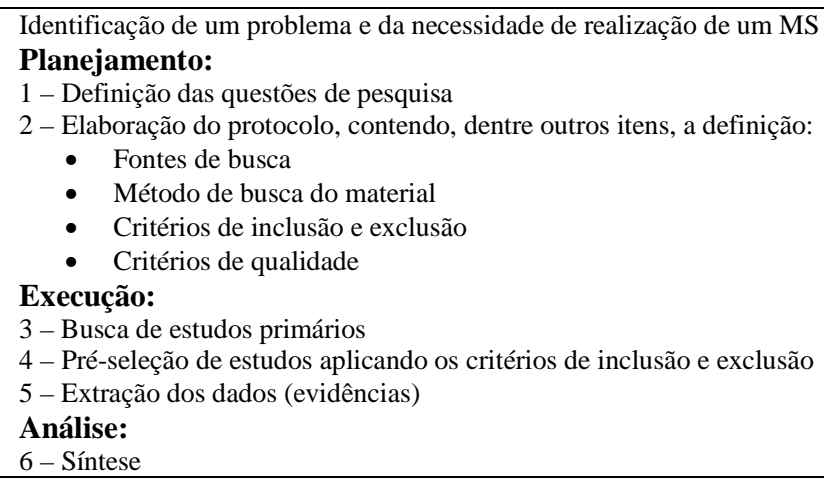




\subsection{Questões de pesquisa}

Uma pergunta principal do tipo exploratória foi definida e, a partir dela, foram levantadas perguntas específicas com o intuito de guiar a coleta e obtenção dos resultados. As perguntas específicas foram classificadas nas seguintes categorias: (1) descritiva, (2) classificativa, (3) relação e efeito e (4) critério de qualidade, sendo estas apresentadas e respondidas nas seções 4 e 5 e a pergunta principal exibida a seguir:

\section{Pergunta Exploratória}

- P1. Quais as iniciativas da TA, que se baseiam no uso de software/hardware e que auxiliam o processo de ensino/aprendizagem?

\subsection{Estratégias de busca}

A estratégia de busca manual foi adotada nesta pesquisa e os artigos analisados pertencem ao acervo do Simpósio Brasileiro de Informática na Educação (SBIE). Foram excluídos previamente todos os artigos dos anos de 1999 e 2000 por não estarem disponíveis para download, resultando em um total de 1149 artigos iniciais.

\subsection{Critérios de inclusão, exclusão e Pré-seleção}

Para a inclusão de uma publicação, seu tema deve ser sobre TA na Educação, como a pergunta principal. Neste mapeamento, foram excluídos os artigos que não estivessem disponíveis para download ou não estivessem escritos em português ou inglês.

Os critérios anteriores foram aplicados aos 1149 artigos obtidos na busca inicial e a pré-seleção resultou em 79 artigos para a próxima fase. O esquema da obtenção destes 79 finais pode ser visto na Figura 1. Esta pré-seleção foi realizada de modo que cada trabalho teve o "título", "resumo”, "palavras-chave” e "conclusão” lidos por dois pesquisadores sem se comunicarem entre si sobre o seu parecer, ao final deste processo, um terceiro pesquisador com o papel de conciliador, mais experiente sobre o assunto identificou as discordâncias e fazendo a leitura dos mesmos tópicos tomou a decisão final pela inclusão ou exclusão do estudo. Esta metodologia de duplas foi empregada tanto nesta como na próxima fase, e visa diminuir o viés da pesquisa.

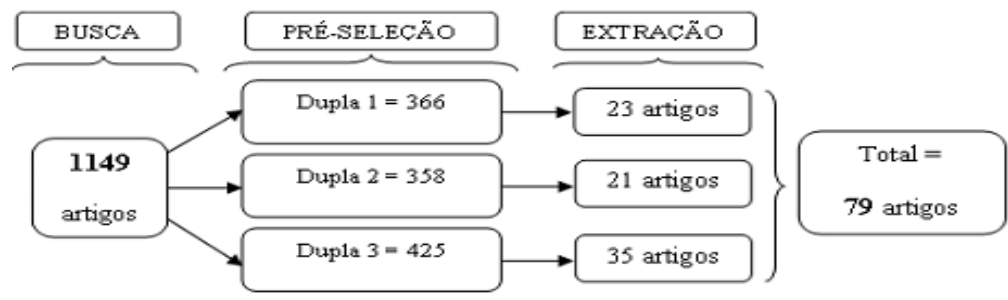

Figura 1. Corpus final após aplicação dos critérios de inclusão e exclusão

\subsection{Extração dos dados e critérios de qualidade}

Nesta fase buscou-se obter informações nos artigos para responder as perguntas de pesquisa. Foi utilizada uma planilha do MS Excel para armazenar e organizar a coleta dos dados. Os dados extraídos de cada um dos 79 artigos foram:

- Ano, tipo do artigo, autores e instituição;

- Classificação do artigo segundo Petersen et al. (2008).

o [1-Pesquisa de validação, 2-Pesquisa de avaliação, 3-Proposta de solução, 4-Artigo filosófico, 5-Artigo sobre opinião, 6-Artigo de 
experiência]

- Quais tipos de software, solução ou estudo;

- Deficiência do estudante;

o [1-Fala, 2-Audição, 3-Visão, 4-Mental/Intelectual, 5-Física/Motora, 6Especificar outra]

- Classificação funcional dos recursos de TA segundo Bersch (2008)

o [1-Auxílios para a vida diária e vida prática, 2-CAA - Comunicação Aumentativa e Alternativa, 3-Recursos de acessibilidade ao computador, 4-Sistemas de controle de ambiente, 5-Projetos arquitetônicos para acessibilidade, 6-Órteses e próteses, 7-Adequação Postural, 8-Auxílios de mobilidade, 9-Auxílios para cegos ou para pessoas com visão subnormal, 10-Auxílios para pessoas com surdez ou com déficit auditivo, 11-Adaptações em veículos]

- Teoria pedagógica;

- Disciplina ou assunto alvo de ensino;

- Modalidade de uso:

o [à distância, presencial ou uso pessoal];

- Se o recurso de TA exige acompanhamento de terceiros;

- Se o recurso de TA exige adaptação de hardware ou de outra tecnologia;

- Tecnologia(s) utiliza(s);

- Como foi feita a validação / avaliação.

\subsection{Síntese dos dados}

Uma vez levantados os dados, o passo seguinte envolveu a interpretação das passagens retiradas dos estudos selecionados a fim de responder as perguntas de pesquisa. Esta é uma fase um tanto subjetiva e exige a experiência dos envolvidos em identificar as respostas semelhantes que usaram termos sinônimos, o que pode gerar muitas discussões internas. Estes dados foram tabulados, gerado as formas de apresentação dos resultados em gráficos e tabelas, além disso, foram mapeadas as evidências dos estudos selecionados.

\section{Resultados}

Os resultados das perguntas intermediárias do MS realizado na literatura do SBIE tratando sobre TA são apresentados nesta seção. Por questões de espaço, a lista dos 79 estudos primários está disponível online ${ }^{2}$. Para cada artigo, foi atribuído um código de identificação que finaliza com 'c' para artigos completos e ' $r$ ' para os resumos expandidos.

\subsection{Respostas às perguntas de pesquisa}

\section{- Perguntas Descritivas:}

\section{P1.1. Qual a evolução do número de trabalhos publicados?}

A Figura 2 ilustra os estudos analisados nessa revisão agrupados por ano de publicação, entre 2001 e 2012 e evidencia que o número de publicações é crescente. O ano de 2001 teve apenas um trabalho na área, em contrapartida o seu pico foi atingido em 2007 com um total de doze artigos, nos anos seguintes há uma diminuição de publicações, mas em 2010 esse número volta a crescer atingindo novamente a marca de

\footnotetext{
${ }^{2}$ Disponível em: http://bit.ly/14WuoKg
} 
doze artigos todos eles completos em 2012.

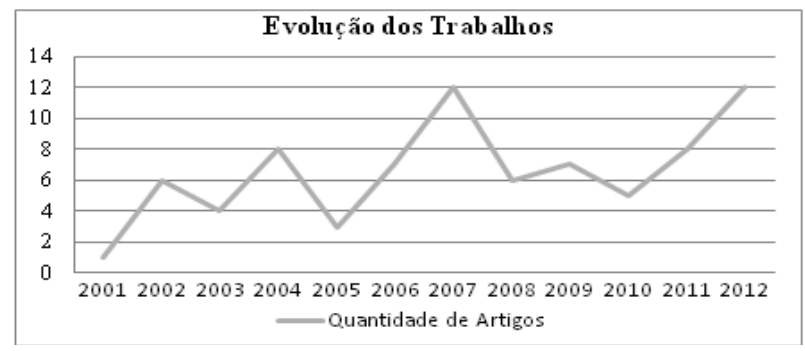

Figura 2. Evolução dos Trabalhos

\section{P1.2. Quais são os grupos de pesquisa atuantes nesta área?}

A Tabela 1 mostra como se distribuiu o número de publicações por instituição de ensino. A UFRGS se destaca das demais com 13 publicações e mostra um interesse maior em desenvolver TA para apoio a PcD, talvez este resultado se justifique devido ao fato desta universidade ter uma pós graduação em Informática na Educação.

Tabela 1. Instituições e Quantidade de publicações

\begin{tabular}{|l|c|}
\hline \multicolumn{1}{|c|}{ Instituições de Ensino } & Publicações \\
\hline UFRGS & 13 \\
\hline UFRJ & 7 \\
\hline UNICAMP, FEEVALE & 6 \\
\hline UNISINOS, IFRS & 3 \\
\hline UFES, USP, PUCRS, UFPE & 2 \\
\hline $\begin{array}{l}\text { UNIFRA, UNIRIO, Estácio de Sá, UNB, FEEC, UERJ, UNIMEP, ITA, } \\
\text { UNIFEI, ULBRA, UFSCAR, SMED, UNIVALI }\end{array}$ & \\
\hline $\begin{array}{l}\text { UFRPE, UGF, UFAL, Universidade de Coimbra, UNIOESTE, UFOP, FA- } \\
\text { CENSA, IFF, FACOL, UNIME, UFC, UNISC, FEMA, USCS, UFPA, }\end{array}$ & 1 \\
$\begin{array}{l}\text { UTFPR, NTE-SEEDUC, UFAM, AACD, UNIESP, FTT, FATEC, FATEC- } \\
\text { RS, UFMS, IEPPEP, CEFET-ES, NIED, FUOM, Centro Federal de Educa- } \\
\text { ção Tecnológica de Bento Gonçalves, UDESC, UVA, UFPR, IPM }\end{array}$ & \\
\hline
\end{tabular}

Pode-se destacar também os autores com número mais relevante de publicações. Com 9 publicações temos Lucila M. Costi Santarosa, com 6 Débora Conforto, com 4 publicações temos Patrícia B. Scherer Bassani, Sérgio Crespo C. S. Pinto e Vinícius Costa de Souza, com 3 Liliana M. Passerino, Lourenço de Oliveira Basso, Márcia de Borba Campos, Maurício C. Rosito e Regina Heidrich, com 2 publicações foram dezesseis autores e outros 179 publicaram um artigo.

- Perguntas Classificativas:

\section{P1.3. Quais tipos de deficiência são mais assistidos?}

\section{P1.4. Quais não possuem nenhum suporte de TA?}

No presente trabalho foi constatado que todas as deficiências foram citadas, porém enquanto mais da metade das publicações atendem as deficiências de audição e visão, representando ambas $30 \%$ e $28 \%$ respectivamente, apenas $2 \%$ atende a fala. A Tabela 02 mostra estes e os demais resultados incluindo a relação dos trabalhos estudados por tipo de deficiência. No campo “Outras” foram agrupados trabalhos com deficiências muito específicas como, por exemplo, a fibrose cística que incapacita o paciente em alguns períodos e faz com que o mesmo necessite de assistência. 
Tabela 2. Tipos de deficiências mais assistidas

\begin{tabular}{|l|c|l|}
\hline Deficiência & Ocorrência & \multicolumn{1}{|c|}{ Relação de trabalhos } \\
\hline Auditiva & $30 \%$ & $\begin{array}{l}\text { E01c, E06r, E20c, E27c, E28r, E34c, E35r, E36c, E41c, E43c, } \\
\text { E50c, E51c, E52c, E56r, E58c, E59c, E60c, E61r, E63c, E64c, } \\
\text { E68c, E73r, E74r }\end{array}$ \\
\hline Visual & $28 \%$ & $\begin{array}{l}\text { E03c, E08c, E11c, E14r, E15c, E19c, E22c, E23c, E25r, E32r, } \\
\text { E37r, E39r, E40c, E45r, E48c, E54c, E57r, E67c, E71c, E72c, } \\
\text { E76c }\end{array}$ \\
\hline $\begin{array}{l}\text { Mental/ } \\
\text { Intelectual }\end{array}$ & $13 \%$ & $\begin{array}{l}\text { E02c, E07c, E12r, E16r, E24r, E53r, E55c, E69c, E75r, E77c, } \\
\text { E78c }\end{array}$ \\
\hline Múltiplas & $7 \%$ & E04c, E13c, E27c, E47c, E66r, E70r, E79c \\
\hline Motora & $6 \%$ & E10c, E29c, E42c, E65c \\
\hline Fala & $2 \%$ & E05c \\
\hline Outras & $14 \%$ & $\begin{array}{l}\text { E09c, E17r, E18c, E21c, E26c, E30c, E31c, E33c, E38c, E44c, } \\
\text { E46r, E49c, E62c }\end{array}$ \\
\hline
\end{tabular}

Segundo a classificação de Bersch (2008) onde as TA são divididas em onze diferentes tipos, está ilustrada na Figura 3 e pode-se perceber que apenas cinco foram identificadas por este grupo de pesquisa de forma que auxílios para cegos ou para visão subnormal apresentou e auxílios para pessoas com surdez ou com déficit auditivo representaram mais da metade dos estudos, compatível com o resultado da Tabela 2.

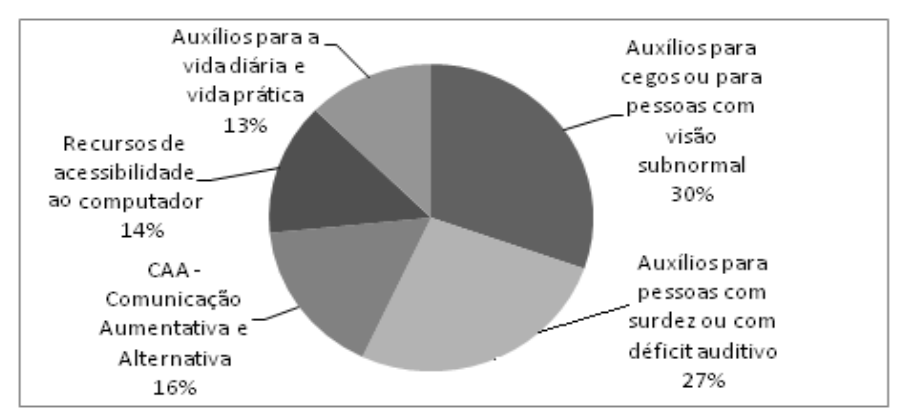

Figura 3. Classificação dos artigos segundo Bersch

\section{P1.5. Quais teorias pedagógicas são empregadas nestes trabalhos?}

Quase a totalidade dos artigos não fundamentou o desenvolvimento do software educativo em uma teoria pedagógica, 70 não citaram o uso de base pedagógica e 1 dos artigos relata o uso de duas simultaneamente. A falta do emprego de uma abordagem pedagógica no desenvolvimento do software para auxílio do aprendizado de PcD evidencia a pouca atenção que os pesquisadores dão a esse quesito e pode influenciar no objetivo final do mesmo que é melhorar ou facilitar o ensino/aprendizagem.

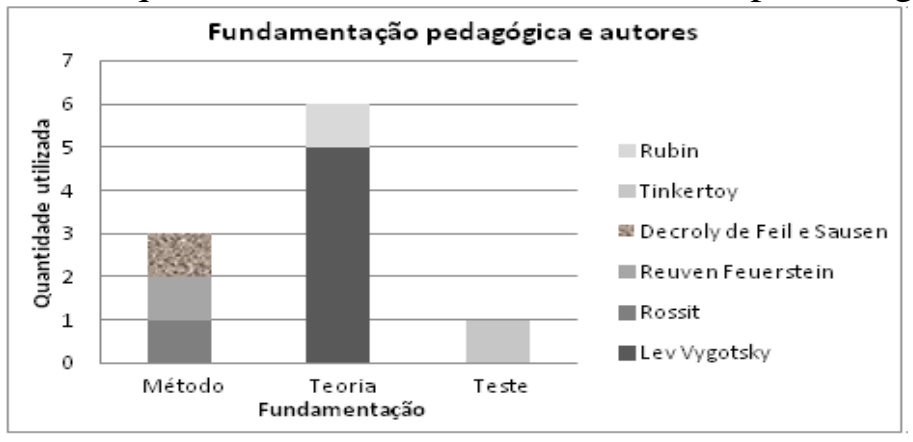

Figura 4. Autores e tipos de fundamentações pedagógicas 
Os criadores das fundamentações estão listados na Figura 4, sendo que estes foram categorizados em método, teoria ou teste. O Mapeamento levantou 6 teorias, 3 métodos e 1 teste, sendo que Vygotsky foi o autor cujo trabalho foi o mais utilizado como base pedagógica, com 5 artigos citando sua teoria.

Sobre as disciplinas ou assunto alvo de ensino, a dificuldade se deveu a alguns trabalhos não deixarem claro qual o objetivo educacional do uso da proposta e também apareceu uma pluralidade de resultados. Porém os mais significativos foram 13 sobre promover a acessibilidade, 9 ensino de libras, 8 alfabetização, 8 facilitar a comunicação e interação, 7 cognição e 6 ensino de informática. Foi percebido também que poucos são os estudos que visam alguma forma de educação à distância, sendo a ênfase no uso presencial e quando se tratava do uso pessoal a maioria das ferramentas foi para o auxilio de atividades diárias, como o uso de navegadores ou mesmo ferramentas físicas.

\section{P1.6. Quais tipos de software, hardware, soluções ou estudos são propostos?}

A análise realizada indicou uma grande variedade de resultados a este quesito, assim optou-se pelo agrupamento destes conforme a Figura 5. Com destaque para produção de outras ferramentas com aproximadamente 51\% de toda a coleta seguindo de AVA 13\% e Jogos 9\%, também as tecnologias usadas na produção foram agrupadas em 12 grupos, com um total de 153 tecnologias catalogadas.

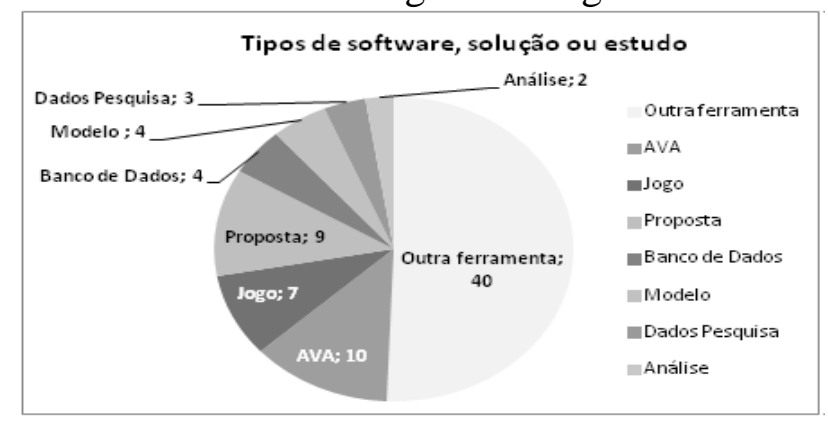

Figura 5. Tipo de software

Percebe-se que, por ser um evento com maior participação de pessoas da área de informática há uma tendência a produção de conteúdo mais prático e usável, em que foi citado 153 tecnologias usadas para produções dos softwares, modelos e estudos propostos, sendo que estes foram agrupados em 12 grupos. Nos estudos foram citadas 41 linguagens de programação, 23 recursos web, 17 banco de dados, 14 ferramentas de acessibilidades complementares, 14 editores de imagem ou texto, 10 padrões de projeto ou algoritmos, 9 dispositivos de hardware, 8 Desktop, 8 ferramentas CAD, 5 player de vídeo e 4 sistemas operacionais.

Apenas 6 software sugeridos nos estudos primários exigiram algum tipo de adaptação de hardware, ajuste ou equipamento especial para a utilização do software desenvolvido, o que representa $8 \%$ do total. A adaptação de hardware nem sempre está associada a deficiências que afetam a atividade motora das pessoas, já que apenas 3 entre os 6 software que devem possuir hardware adaptado são destinados a quem tem o tipo de necessidade sugerida. Software com equipamento adaptado para pessoas com deficiências visual e auditiva também foram desenvolvidos.

\section{P1.7. Qual a classificação do estudo realizado segundo Petersen et al.(2008)?}

No levantamento da classificação do tipo de estudo segundo Petersen (2008) o 
único tipo não identificado foi sobre Opinião. Entre as outras 5 categorias, a mais presente com pouco mais da metade dos trabalhos foi 44 artigos de Propostas de Solução, com isso, nota-se uma preocupação daqueles autores em criar meios alternativos práticos para promover a acessibilidade. As outras categorias ficaram com 24 Pesquisas de Avaliação, 5 Pesquisas de validação, 4 de Experiência e 2 Filosóficos.

\section{Discussão}

Nesta seção são discutidos os resultados obtidos neste mapeamento para a pergunta exploratória, qual a relação e efeito do que foi obtido e a qualidade dos estudos primários para responder a todas as perguntas.

- Pergunta exploratória:

P1. Quais as iniciativas da TA, que se baseiam no uso de software/hardware e que auxiliam o processo de ensino/aprendizagem?

Foram catalogadas 153 tecnologias onde estão incluídas principalmente ferramentas específicas ou adaptações de já existentes, AVA, Jogos, além de teorias, métodos e estudos diversos. Estas tecnologias são oferecidas principalmente para o uso no ambiente de ensino presencial, mas estão disponíveis também opções para o ensino à distância e o uso pessoal e poucas delas necessitam de algum hardware específico.

\section{- Pergunta de Relação e Efeito:}

\section{P2. Qual a implicação das evidências encontradas nos estudos?}

O relato dos autores dos trabalhos estudados citam que o resultados das TA são: 1) facilitação e melhoria da comunicação tornando a PcD melhor integrada ao ambiente; 2) aumento na taxa de retenção do conteúdo abordado; 3) mais facilidade para planejar o ensino, em função da posse de recursos personalizados. Logo, esta é uma área que vale a pena investir esforços em pesquisa e aplicação.

\section{- Perguntas de Critérios de qualidade - força da evidência:}

\section{P3. De que forma os estudos primários foram validados/avaliados?}

Embora os estudos primários selecionados para esta fase fossem todos aprovados pelas bancas do evento, esta seleção teve a sua qualidade avaliada para este mapeamento. Esta não foi um critério de exclusão de estudos, foi apenas constado se a força de que a evidência estudada realmente é aplicável e tem resultado positivo.

Sobre os 44 estudos com Proposta de solução foi constado que apenas 17 afirmaram ter realizado validação daquela solução. Felizmente quando o trabalho tinha a descrição do perfil do usuário testado, estes sempre representavam o público alvo a que se destina a solução. Porém nos estudos primários classificados especificamente como Pesquisa de validação ou de Avaliação (9) alguns não tiveram esta preocupação e utilizaram usuários de outras categorias como programadores ou estudantes comuns.

\section{Conclusão}

No MS realizado neste trabalho o grupo concluiu que em P2 e P3 houve muitos relatos de contribuições necessárias e importantes para o bom andamento do processo de ensino/aprendizagem de PcD, incapacidades ou mobilidade reduzida e os problemas encontrados estavam passando por mais testes ou já em fase de melhorias, o que é positivo. Porém quando se trata de avaliações e validações a resposta a P3 evidencia que 
os autores devem se preocupar mais com este tópico tão importante, pois somente a escolha de uma amostra significativa e um experimento bem elaborado e realizado garante que o trabalho realmente alcançou seu objetivo e obteve a contribuição descrita.

Também foi concluído, que assim como a informática está cada vez mais presente na educação, ela continua criando personalizações, e está atendendo a grupos distintos como o deste estudo, porém o resultado de P1 evidenciou que embora todas as deficiências sejam assistidas pelos estudos, enquanto as deficiências mais numerosas na população brasileira são a visual seguida pela motora, os estudos se concentram na auditiva e visual.

Como trabalho futuro é sugerido fazer um aprimoramento dos resultados obtidos na área de tecnologias usadas (P1.6), pois estas informações além de mostrar a tendência de uso, ainda pode nortear grupos de pesquisa, que são iniciantes em TA.

\section{Referências bibliográficas}

Bersch, Rita. (2008) Introdução à Tecnologia Assistiva. CEDI - Centro Especializado em Desenvolvimento Infantil. Porto Alegre/RS. Disponível em: < http://www.assistiva.com.br/tassistiva.html\#categorias>. Acessado em: 15/07/2013.

Biolchini, J.; Mian, P. G., Natali, A. C. C. and Travassos, G. H. (2005) Systematic Review in Software Engineering. Technical Report, RT - ES679/05. Systems Engineering and Computer Science Department. COPPE/UFRJ.

Comitê de Ajudas Técnicas - CAT (2009) Tecnologia Assistiva. Subsecretaria Nacional de Promoção dos Direitos da Pessoa com Deficiência, CORDE, Brasília.

Dyba, T.; Kitchenham, B.A.; Jorgensen, M. (2005) Evidence-based software engineering for practitioners. Software, IEEE, vol.22, no.1, pp.58,65.

European Commission - DGXIII. (2008). Critical factors involved in end-users' education in relation to Assistive Technology. Disponível em: http://www.siva.it/research/eustat/download_eng.htm. Acessado em: 15/07/2013.

ISO 9999 (2007) Assistive products for persons with disability - Classification and terminology. International Organization for Standardization. Fourth edition.

Kitchenham, B.; Dybå, T.; Jørgensen, M. (2004) Evidence-based Software Engineering. 26th International Conference on Software Engineering, (ICSE '04), Proceedings. IEEE, Washington DC, USA, pp 273 - 281.

Ministério Público Federal: Fundação Procurador Pedro Jorge de Melo e Silva (2004). Cartilha Acesso de Alunos com Deficiência às Escolas e Classes Comuns da Rede Regular. $2^{\mathrm{a}}$ ed. rev. e atualiz., Procuradoria Federal dos Direitos do Cidadão, Brasília.

Petersen, Kai; Feldt, Robert; Mujtaba, Shahid; Mattsson, Michael. (2008) Systematic Mapping Studies in Software Engineering. Proceeding EASE'08 - 12th international conference on Evaluation and Assessment in Software Engineering, pp 68-77.

Program Technology Initiative for Disabled and Elderly People - TIDE (1998) Horizontal European Activities in Rehabilitation Technology. European Commission - DGXIII.

USA - Department of Education - National Classification System (2000). Disponível em: www.ed.gov/offices/OSERS/NIDRR/Products/National_Classification_System.do. 